LA-UR-09- 06529

October 7, 2009

Approved for public release:

distribution is unlimited.

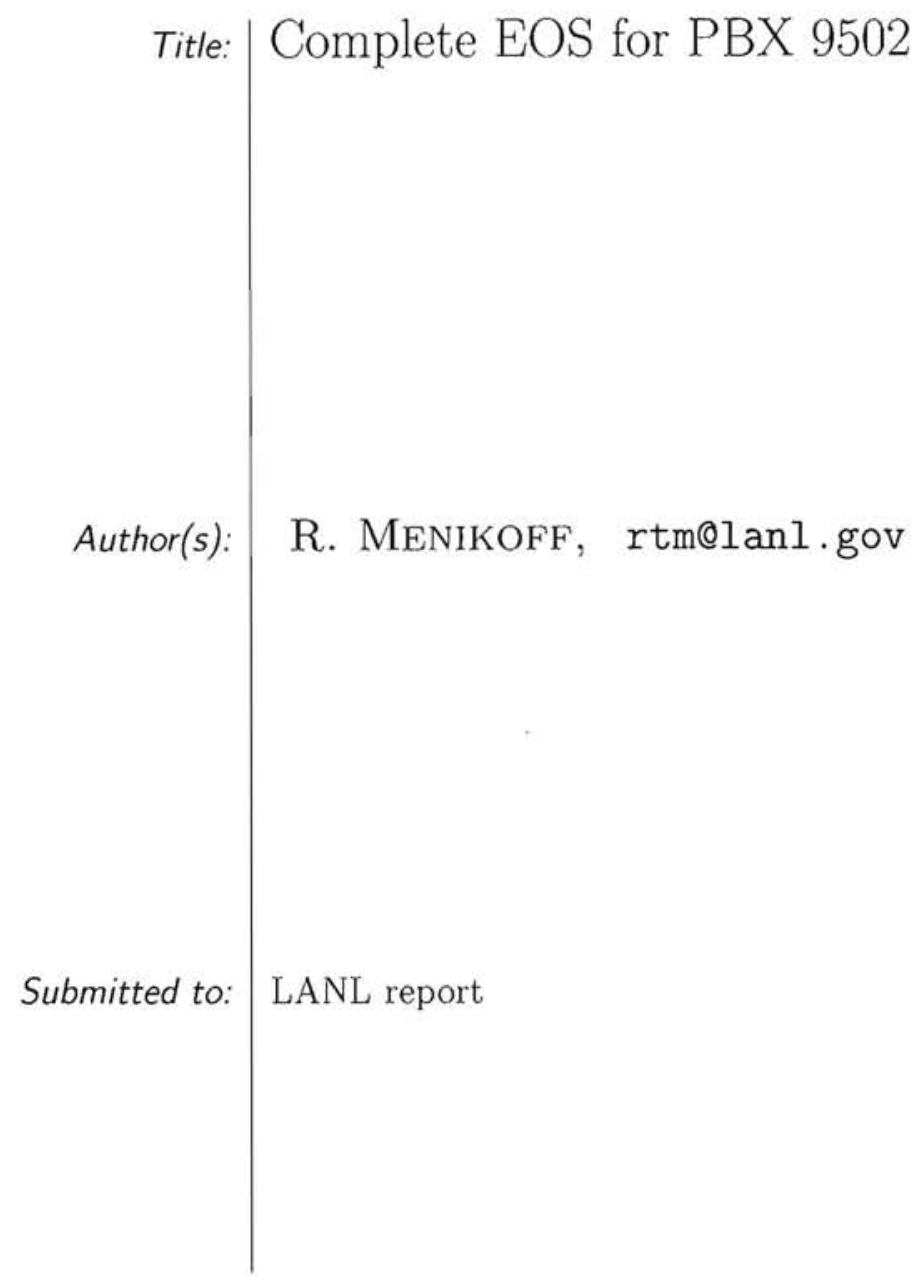

- Los Alamos NATIONAL LABORATORY EST.1943

Los Alamos National Laboratory, an affirmative action/equal opportunity employer, is operated by the Los Alamos National Security, LLC for the National Nuclear Security Administration of the U.S. Department of Energy under contract DE-AC52-06NA25396. By acceptance of this article, the published recognizes that the U.S. Government retains a nonexclusive, royalty-free license to publish or reproduce the published form of this contribution, or to allow others to do so, for U.S. Government purposes. Los Alamos National Laboratory requests that the publisher identify this article as work performed under the auspices of the U.S. Department of Energy. Los Alamos National Laboratory strongly supports academic freedom and a researcher's right to publish; as an institution, however, the Laboratory does not endorse the viewpoint of a publication or guarantee its technical correctness. 



\title{
Complete EOS for PBX 9502
}

\author{
Ralph Menikoff*
}

October 7, 2009

\begin{abstract}
PBX 9502 is an insensitive plastic-bonded explosive based on triaminotrinitrobenzene (TATB). A complete equation of state (EOS) is constructed for unreacted PBX 9502 suitable for reactive burn models, i.e., high pressure regime in which material strength is unimportant. The PBX EOS is composed of two parts: a complete EOS for TATB and a porosity model which allows for variations in the initial PBX density. The TATB EOS is based on a cold curve and a thermal model for lattice vibrations. The heat capacity, and hence thermal model, is determined by the vibrational spectrum from Raman scattering. The cold curve is calibrated to diamond anvil cell data for isothermal compression using a two-piece Keane fitting form. Hugoniot data for PBX 9502 is used as a consistency check.
\end{abstract}

\section{Introduction}

PBX 9502 is an insensitive high explosive. It consists of $95 \mathrm{wt} \%$ TATB grains held together by $5 \mathrm{wt} \% \mathrm{Kel}-\mathrm{F}$ binder. In addition, there is a small amount of porosity due to the pressing process used to manufacture a PBX. At its nominal density, $1.895 \mathrm{~g} / \mathrm{cm}^{3}$, PBX 9502 has a porosity of $2.4 \%$.

Reactive burn models require equations of state (EOS) for both the reactants and the products of an explosive. Typically, reaction models for

*e-mail: rtm@lanl.gov 
heterogeneous explosives, such as Ignition \& Growth [1], use a pressure dependent reaction rate. For models of this class, the reaction is insensitive to the thermal properties of the reactants. Consequently, a reactant EOS with a constant specific heat can be used, even though, explosives are large molecules for which the specific heat varies with temperature.

A notable recent reaction model, CREST [2], takes the reaction rate to be a function of the reactant entropy. It aims to describe shock initiation of a PBX over a range of initial densities. For such a model, a complete EOS is needed for the reactants which accounts for the initial PBX porosity.

Here a thermodynamically consistent EOS is constructed for the reactants of PBX 9502. It is composed of a complete EOS for the PBX at theoretical maximum density (TMD) and a porosity model. (We note that Lambourn et al. [3] used a similar approach.) Since PBX 9502 is predominantly TATB, pure TATB is used to approximate the PBX at TMD. We expect this approximation to result in a slightly stiffer EOS since the TATB grains are crystalline whereas the Kel-F binder is an amorphous polymer.

A generalized Hayes EOS is used for the TATB EOS. The formulation of this EOS is reviewed in sec. 2. Fitting forms for the cold curve and heat capacity suitable for TATB are described in sec. 3. The porosity model used for the PBX is reviewed in sec. 4. Model parameters for the cold curve are fit to isothermal compression data from diamond anvil cell experiments, and the specific heat is fit to lattice vibrational frequencies from Raman scattering data. The calibration for TATB is described in sec. 5. As a check on the model, in sec. 6 we compare with shock Hugoniot data for PBX 9502.

Section 7 is a summary of the EOS model for PBX 9502 in which some of its limitations are discussed. The two largest inaccuracies stem from neglecting the temperature dependence of the Grüneisen coefficient, and from the simplifying approximation for the expansion region below the initial density. Nevertheless, the EOS presented here is an improvement over what is currently used for modeling reactive flow in PBX 9502.

\section{Generalized Hayes EOS}

The generalized Hayes EOS (see [4, sec. 4.3.4] and [5]) is of the Mie-Grüneisen type. It is thermodynamically consistent and allows for a non-constant spe- 
cific heat. Previously, it has been applied to the explosive HMX [6]. We will use this EOS form for TATB.

The generalize Hayes EOS is defined by the Helmholtz free energy

$$
F(V, T)=e_{0}-\int_{V_{0}}^{V} P_{c}\left(V^{\prime}\right) \mathrm{d} V^{\prime}-\int_{0}^{T}\left(T-T^{\prime}\right) \tilde{C}\left(T^{\prime} / \theta(V)\right) \frac{\mathrm{d} T^{\prime}}{T^{\prime}},
$$

where $\left(V_{0}, e_{0}\right)$ is a reference point on the cold curve, $P_{c}(V)$ is the pressure on the cold curve, i.e., at $T=0, \tilde{C}(\tilde{T})$ is a specific heat and $\theta(V)$ is a temperature scale. The specific heat at constant volume is required to have the property

$$
C_{V}(V, T)=C_{V}\left(V_{0}, \theta\left(V_{0}\right)[T / \theta(V)]\right)=\tilde{C}(\tilde{T}),
$$

i.e., $C_{V}$ is a function of only the scaled temperature $\tilde{T}=T / \theta(V)$. Hence, the temperature dependence of the specific heat at $V_{0}$, i.e.,

$$
C_{V}\left(V_{0}, T\right)=\tilde{C}\left(T / \theta\left(V_{0}\right)\right)
$$

and $\theta(V)$ completely determine the specific heat.

Moreover, we require Nernst law to hold, i.e., $\tilde{C}(\tilde{T}) \rightarrow 0$ as $\tilde{T} \rightarrow 0$, in order for the second integral on the right hand side of Eq. (1a) to be well defined. This implies that the cold curve is both an isotherm and an isentrope.

Using the standard thermodynamic identities, it can be shown that the pressure can be expressed in the Mie-Grüneisen form

$$
P(V, e)=P_{c}(V)+\frac{\Gamma(V)}{V}\left[e-e_{c}(V)\right],
$$

where the energy on the cold curve is

$$
e_{c}(V)=e_{0}-\int_{V_{0}}^{V} P_{c}(V) \mathrm{d} V,
$$

and the Grüneisen coefficient is

$$
\Gamma(V)=-\frac{\mathrm{d} \ln \theta(V)}{\mathrm{d} \ln V} .
$$


Thus, the temperature scale $\theta(V)$ plays a role similar to a "Debye temperature."

By using the specific heat, the energy can be expressed in terms of the temperature

$$
e(V, T)=e_{c}(V)+\int_{0}^{T} \tilde{C}\left(T^{\prime} / \theta(V)\right) \mathrm{d} T^{\prime},
$$

which can be inverted to obtain $T(V, e)$. The corresponding entropy is

$$
S(V, e)=\int_{0}^{T(V, e)} \tilde{C}\left(T^{\prime} / \theta(V)\right) \frac{\mathrm{d} T^{\prime}}{T^{\prime}} .
$$

Since the thermodynamic variables are derived from a free energy, the thermodynamic identity, $\mathrm{d} e=-P \mathrm{~d} V+T \mathrm{~d} S$, is satisfied.

We note that Lambourn et al. [3] have constructed an EOS for TATB. Though formulated differently, their form of EOS is equivalent to the free energy and specific heat given by Eq. (1). As applied to TATB, we will use different fitting forms for the reference curve (cold curve vs. principal isentrope) and the specific heat. We also use a different calibration procedure.

\section{Fitting forms}

The generalized Hayes EOS is determined by three functions; $P_{c}(V), \theta(V)$ and $\tilde{C}(T / \theta(V))$. Here we describe the fitting forms for these functions that we will use to construct an EOS for TATB.

\subsection{Cold curve}

The Keane 'EOS' is a convenient fitting form for the cold curve. It is defined by a relation between the bulk modulus $K=-V(\mathrm{~d} P / \mathrm{d} V)_{T=0}$ and its derivative $K^{\prime}=\mathrm{d} K / \mathrm{d} P$, see [7] and references therein,

$$
K^{\prime}=K_{\infty}^{\prime}+\frac{\left(K_{0}^{\prime}-K_{\infty}^{\prime}\right) K_{0}}{K},
$$

where subscripts ' 0 ' and ' $\infty$ ' denote values at $P=P_{0}$ and $\infty$, respectively. Equation (5) can be integrated to yield

$$
\frac{K(V)}{K_{0}}=1+\frac{K_{0}^{\prime}}{K_{\infty}^{\prime}}\left[\left(\frac{V}{V_{0}}\right)^{-K_{\infty}^{\prime}}-1\right]
$$


and then again to obtain

$$
\frac{P_{c}(V)-P_{0}}{K_{0}}=\frac{K_{0}^{\prime}}{K_{\infty}^{\prime 2}}\left[\left(\frac{V}{V_{0}}\right)^{-K_{\infty}^{\prime}}-1\right]+\left(\frac{K_{0}^{\prime}}{K_{\infty}^{\prime}}-1\right) \ln \left(\frac{V}{V_{0}}\right) .
$$

We note that the Keane pressure is defined by a reference state $\left(V_{0}, P_{0}\right)$ and three parameters; $K_{0}, K_{0}^{\prime}$ and $K_{\infty}^{\prime}$. Due to Eq. (5), $P_{c}(V)$ is independent of the reference point, provided that $K_{0}$ and $P_{0}$ are evaluated at $V_{0}$.

To fit TATB we will use a piecewise Keane EOS, i.e., $P_{c}(V)$ is given by Eq. (7) with parameters $\left(V_{0}, P_{0}\right), K_{0}, K_{0}^{\prime}$ and $K_{0, \infty}^{\prime}$ for $V_{1} \leq V \leq V_{0}$ and parameters $\left(V_{1}, P_{1}\right), K_{1}, K_{1}^{\prime}$ and $K_{1, \infty}^{\prime}$ for $V<V_{1}$. By choosing $P_{1}=P_{c}\left(V_{1}\right)$ and $K_{1}=K\left(V_{1}\right)$ with the first set of parameters, both the pressure and the bulk modulus are continuous. This adds three more parameters $\left(V_{1}, K_{1}^{\prime}\right.$ and $\left.K_{1, \infty}^{\prime}\right)$, and gives added flexibility to fit kinks in isothermal and Hugoniot data.

Physically, the discontinuity in $K^{\prime}$ can be motivated as follows. A TATB crystal has a layered structure similar to that in graphite, but with carbon atoms replaced by TATB molecules. In the plane of each layer, the crystal is very stiff. Consequently, at low pressures, compression is predominantly between layers. When the layers get close enough to feel the atomic repulsion, the response to further compression changes. We note that a phase transition has been observed in graphite at room temperature in which the layer spacing changes discontinuously [8, fig. 2]. (Very likely, hexagonal to rhomboidal structure rather than the graphite-diamond transition.) The graphite transition pressure, about $11 \mathrm{GPa}$, is close to the kink in diamond anvil cell data for TATB at about $8 \mathrm{GPa}[9$, fig. 7$]$ and also in the Hugoniot data for PBX 9502 [10, fig. 4]. In addition, Raman scattering data of TATB shows a kink in the high frequency vibrational modes [11, fig. 4a] associated with the coupling between crystal layers. Also, a change in the color of TATB crystal with pressure has been observe in diamond anvil cell experiments [12, 9]. This is indicative of a change in electron structure or bonding of molecules in the crystal, see discussion in [9, sec. 4.3].

A discontinuity in $K^{\prime}$ corresponds to a discontinuity in the third derivative of the free energy. Technically, it would corresponds to a third-order phase transition. Since the pressure and sound speed are continuous, there would be no qualitative change in the wave structure like the wave splitting associated with a first-order phase transition. Thus, the discontinuity is very weak and not likely to affect hydro simulations. 


\subsection{Thermal model}

Explosives are large molecules and have many vibrational modes. For example, a TATB molecule $\left(\mathrm{C}_{6} \mathrm{H}_{6} \mathrm{~N}_{6} \mathrm{O}_{6}\right)$ has 24 atoms and 72 modes. In the detonation regime, roughly $P<50 \mathrm{GPa}$ and $T<5000 \mathrm{~K}$, solid explosives are electrically non-conducting. Consequently, their specific heat is dominated by lattice vibrations or optical phonons. The vibrational frequencies, $\nu_{i}$, can be determined from Raman scattering and infrared spectrometry, or from the Fourier spectrum of the velocity auto-correlation computed with molecular dynamics. In general, the frequencies are a function of $V$.

Treating each phonon as a quantum oscillator (quasi-harmonic approximation), the specific heat is given by

$$
C_{V}(V, T)=\sum_{i} \hat{C}\left(T / \theta_{i}(V)\right),
$$

where $k_{B} \theta_{i}(V)=h \nu_{i}(V), k_{B}$ is the Boltzmann constant, and $h$ is the Planck constant. The mode specific heat is

$$
\hat{C}\left(x^{-1}\right)=\frac{R}{\mathrm{~mol} w \mathrm{t}} \cdot\left[\frac{x}{\exp (x)-1}\right]^{2} \exp (x),
$$

where $x=T / \theta=k_{B} T /(h \nu)$ and $R$ is the gas constant $(8.314 \mathrm{~J} / \mathrm{mol} \cdot \mathrm{K})$. The molecular weight of TATB is $258.18 \mathrm{~g} / \mathrm{mol}$. A plot of the mode specific heat is shown in fig. 1 . We note that a mode is half saturated at $T \approx \frac{1}{3} \theta_{i}$ and $90 \%$ saturated at $T \approx 0.9 \theta_{i}$. We also note that $\theta /[\mathrm{K}]=1.44 \nu /\left[\mathrm{cm}^{-1}\right]$. Thus, at room temperature $(300 \mathrm{~K})$, modes with frequencies $\nu<625 \mathrm{~cm}^{-1}$ would have $\theta<900 \mathrm{~K}$, be half saturated and contribute significantly to the specific heat.

For each phonon, one can define a mode Grüneisen coefficient

$$
\Gamma_{i}(V)=-\mathrm{d} \ln \theta_{i}(V) / \mathrm{d} \ln V .
$$

In general the Grüneisen coefficient is the specific heat weighted sum of the mode coefficients, i.e.,

$$
\Gamma(V, T)=\frac{\sum_{i} \Gamma_{i}(V) \hat{C}\left(T / \theta_{i}(V)\right)}{\sum_{i} \hat{C}\left(T / \theta_{i}(V)\right)} .
$$

Consequently, $\Gamma$ is a function of both $V$ and $T$. As the temperature increases, the high frequency modes are weighted more heavily. Typically, the high frequencies have a lower mode $\Gamma_{i}$, and as a result $\Gamma$ decreases with increasing $T$. 


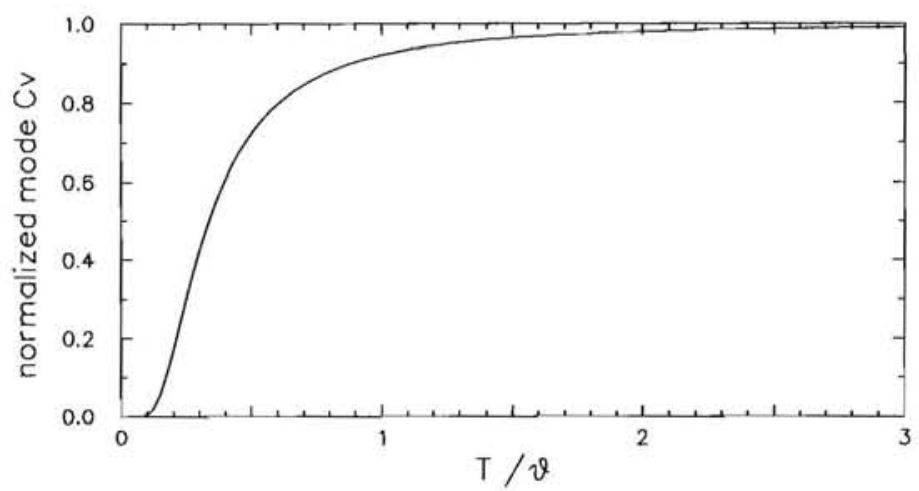

Figure 1: Normalized specific heat for each mode Eq. (8b).

The free energy for the generalized Hayes EOS, Eq. (1), assumes only one temperature scale, i.e., $\theta_{i}(V)=\theta(V)$ for all $i$. As a consequence, the Grüneisen coefficient reduces to a function of only $V$. For a large class of applications, it is most important for the EOS to be accurate in the vicinity of the principal Hugoniot locus. Since the temperature increases with shock strength, we expect the Grüneisen coefficient to decrease with shock compression, i.e., $\Gamma(V)$ decreases with decreasing $V$ on the Hugoniot locus. Due to the lack of data, we use the simple fitting form

$$
\Gamma(V)=\Gamma_{0}+\Gamma_{1}\left(V / V_{0}\right) \text {. }
$$

Then for the temperature scale, we need

$$
\theta(V)=\theta_{0}\left(V_{0} / V\right)^{\Gamma_{0}} \exp \left[\Gamma_{1}\left(1-V / V_{0}\right)\right] .
$$

The coefficient $\theta_{0}$ is not important provided that it is used consistently between Eqs. (10b) and (2) when calibrating the specific heat function $\tilde{C}(\tilde{T})$. We will use it merely to set the unit for temperature and take $\theta_{0}=1 \mathrm{~K}$.

It can be shown from the general properties of the mode specific heat, Eq. (8b), and the acoustic phonon spectrum (Debye model) that the specific heat has the limiting properties

$$
\tilde{C}(\tilde{T}) \propto \begin{cases}\tilde{T}^{3}, & \text { as } \tilde{T} \rightarrow 0 ; \\ \text { constant }, & \text { as } \tilde{T} \rightarrow \infty\end{cases}
$$


A fitting form that has this asymptotic behavior is

$$
\tilde{C}(\tilde{T})=\frac{C_{v} \tilde{T}^{3}}{c_{0}+c_{1} \tilde{T}+c_{2} \tilde{T}^{2}+c_{3} \tilde{T}^{3}} .
$$

The coefficient $C_{v}$ has units of specific heat while the $c_{i}$ are dimensionless. We will use this simple form, though one could do better with a cubic spline.

\subsection{Extension to expansion regime}

Though solid EOS, like the one described in this section, are designed for the compression regime, $V \leq V_{0}$, some applications require evaluating the pressure in the expansion regime, $V>V_{0}$. For example, if a detonation wave impinges on a free surface or at the boundary of an unconfined rate stick, then the rapid pressure drop can cause hot reactants within the reaction zone to expand to low density. This can cause problems with reaction models, such as those that use a pressure-temperature equilibrium mixing rule for a partly burned explosive. Pressure-temperature equilibrium of the reactants and products only has a unique solution if each component EOS is thermodynamically stable, i.e., both the isothermal compressibility and the specific heat at constant volume are positive. Typically, for a relatively small expansion $\left(V / V_{0}\right.$ slightly greater than 1$)$, the isothermal compressibility of a solid EOS model becomes negative.

In the context of an equilibrium single phase solid EOS, a workaround to ensure thermodynamic stability requires two modifications for $V>V_{0}$ : (i) a cutoff on $P_{c}(V)$, and (ii) limiting the value of $\Gamma(V)$. Physically, the single phase assumption may break down due to spall or sublimation that results in a phase separated mixed solid-gas region. Nevertheless, extending the domain of a solid EOS is useful for robustness of hydro simulations in which only a small localized region of the flow undergoes expansion.

For a Mie-Grüneisen EOS, Eq. (3a), it can be shown that the isothermal compressibility can be written as

$$
K_{T}=-V \frac{\mathrm{d} P_{c}}{\mathrm{~d} V}+\frac{\Gamma^{2}}{V}\left[e-e_{c}(V)-T C_{V}(T, V)\right]-V \frac{\mathrm{d}}{\mathrm{d} V}\left(\frac{\Gamma}{V}\right) \cdot\left[e-e_{c}(V)\right] .
$$

To ensure $K_{T}$ is positive, it is sufficient to make each term on the right hand side (RHS) of Eq. (13) positive. First, we find the minimum pressure on the 
cold curve fitting form, i.e., $V_{*}$ such that $\mathrm{d} P_{c} / \mathrm{d} V\left(V_{*}\right)=0$. Then for $V>V_{*}$, we replace the fitting form, Eq. ( 7 ) and Eq. (3b), with a cutoff form

$$
\begin{aligned}
& P_{c}(V)=P_{c}\left(V_{*}\right), \\
& e_{c}(V)=e_{c}\left(V_{*}\right)-P_{c}\left(V_{*}\right)\left(V-V_{*}\right) .
\end{aligned}
$$

This guarantees that the first term on the RHS of Eq. (13) is non-negative. The minimum pressure $P_{c}\left(V_{*}\right)$ will be negative. The model is not meant for tension but rather for temperatures sufficiently high that the thermal pressure results in the total pressure, Eq. (3a), being positive.

For the second term on the RHS of Eq. (13), the factor in brackets can be expressed using Eq. (4a) as

$$
e-e_{c}(V)-T C_{V}(T, V)=\int_{0}^{T} \tilde{C}\left(T^{\prime} / \theta(V)\right) \mathrm{d} T^{\prime}-T \tilde{C}(T / \theta(V)) .
$$

Except for very low $T$, figs. 1 and 2 show that $\tilde{C}(\tilde{T})$ is concave down. Hence, the RHS of Eq. (15) is positive in the regime of interest; room temperature and above.

Next, we modify $\Gamma$ to be

$$
\frac{\Gamma(V)}{V}= \begin{cases}\frac{\Gamma_{0}}{V}+\frac{\Gamma_{1}}{V_{0}}, & \text { for } V \leq V_{0} \\ \frac{\Gamma_{0}}{V}+\frac{\Gamma_{1}}{\left[V_{0}^{2}+\left(V-V_{0}\right)^{2} / \epsilon_{1}^{2}\right]^{1 / 2}}, & \text { for } V>V_{0}\end{cases}
$$

where $\epsilon_{1}$ is a parameter that controls how fast $\Gamma$ approaches a constant with increasing $V$. It is easily verified that both $\Gamma / V$ and its derivative are continuous at $V_{0}$, and that $(\mathrm{d} / \mathrm{d} V)(\Gamma / V)<0$ for all $V$ and any $\Gamma_{0}, \Gamma_{1} \geq 0$. Consequently, the third term on the RHS of Eq. (13) is positive.

Finally, we modify the temperature scale to be consistent with Eq. (3c):

$$
\frac{\theta(V)}{\theta_{0}}= \begin{cases}\left(V / V_{0}\right)^{\Gamma_{0}} \exp \left[-\Gamma_{1} \eta\right], & \text { for } V \leq V_{0} \\ \left(V / V_{0}\right)^{\Gamma_{0}} \exp \left[-\Gamma_{1} \epsilon_{1} \ln \left(\frac{\eta+\left(\epsilon_{1}^{2}+\eta^{2}\right)^{1 / 2}}{\epsilon_{1}}\right)\right], & \text { for } V>V_{0}\end{cases}
$$

where $\eta=V / V_{0}-1$. The result of these modifications is that the EOS model is well behaved in expansion: $K_{T}>0$ and $\Gamma \rightarrow$ constant for large $V$. Moreover, $\Gamma$ and $K_{T}$ are continuous at $V_{0}$. 


\section{Porosity model}

We base the thermodynamic properties of a porous material on a free energy of the form

$$
F(V, T, \phi)=F_{s}(\phi V, T)+B(\phi),
$$

where $\phi$ is the solid volume fraction (1-porosity), $F_{s}$ is the free energy of the pure solid, and $B$ is a potential energy for compaction. It follows that the material pressure,

$$
P(V, T, \phi)=-\partial_{V} F=\phi P_{s}(\phi V, T),
$$

has the same form as that used in the $P-\alpha$ model; see [13], [4, sec. 4.5] and references therein.

The equilibrium condition, $\partial_{\phi} F=0$, is

$$
V P=\phi \frac{\mathrm{d} B}{\mathrm{~d} \phi} .
$$

Alternatively, the equilibrium volume fraction is a function of $V P$. This function is called a compaction law. For simplicity, we use the simple fitting form

$$
\phi_{\mathrm{eq}}(V P)=1-\left(1-\phi_{0}\right) \exp \left[-\frac{V P-V_{0} P_{0}}{V_{s 0} P_{\mathrm{cr}}}\right],
$$

where the subscript ' 0 ' denotes the initial state and $P_{\mathrm{cr}}$ is a parameter.

The parameter $P_{\mathrm{cr}}$ is called the crush-up pressure. For $P>P_{\mathrm{cr}}$, the pores are nearly all squeezed out, $\phi \approx 1$, and the material pressure reduces to that of the pure solid. This limit correspond to the 'snow-plow' model. We expect $P_{\text {cr }}$ to be comparable to the yield strength at which grains deform plastically.

Several points are noteworthy:

(i) A small amount of porosity can significantly lower the initial sound speed. This in turn gives the Hugoniot locus in the $\left(u_{p}, u_{s}\right)$-plane a characteristic concave downward shape in the neighborhood of the initial state.

(ii) Typically, $P_{\mathrm{cr}}$ is much less than the bulk modulus. Consequently, when the pores are being compressed, $V_{s} \approx V_{s 0}$, and the compaction law can be approximated as

$$
\phi_{\mathrm{eq}}\left(P_{s}\right)=1-\left(1-\phi_{0}\right) \exp \left[-\frac{P_{s}-P_{s 0}}{P_{\mathrm{cr}}}\right] .
$$

(iii) Compaction of a PBX is irreversible when unloading from $P>P_{\mathrm{cr}}$. This can be accounted for by only allowing $\phi$ to increase. 


\section{Calibration to TATB}

To construct a TATB EOS we need to determine three functions; $\tilde{C}(\tilde{T}), \theta(V)$ and $P_{c}(V)$. The best available data comes from the Raman spectrum and diamond anvil cell experiments.

\subsection{Specific heat \& Grüneisen coefficient}

For TATB the entire Raman spectrum has been measured over a range of temperatures by McGrane and Shreve [14]. Though many lattice frequencies have been identified, there are two issue with determining all modes. First, a TATB crystal is triclinic and has two molecules per unit cell. This doubles the number of vibrational modes, which equals $3 \times$ (number of atoms per unit cell). Most modes are associated with intra-molecular vibrations and would be doubly degenerate or have a small splitting. But some modes, mainly low frequencies, associated with both intra- and inter-molecular vibrations, would be distinct. Second, selection rules due to molecular and crystal symmetry exclude some modes from the Raman spectrum. Only 85 out of 144 distinct frequencies have been found in the spectrum and listed in [14, table 1].

To help identify the modes, density functional theory calculations have been performed by Liu et al. [15]. Their table 2 has identified 59 modes. Since low frequency modes are hard to calculate, we have supplemented these with 10 low frequency modes from [14, table 1]. The contribution of the low frequency modes to $C_{V}$ is fully saturated above room temperature, so their precise values are not critical. In addition, we assume that the 3 acoustic modes are fully saturated by room temperature. This adds up to 72 modes or $3 \times$ ( 24 atoms per molecule), which is sufficient for a good estimate of the specific heat.

Using the frequencies listed in table 1, the specific heat is computed from Eq. (8). Computed points along with a fit using Eq. (12) are shown in fig. 2. We note that the six highest frequencies (3300 to $3400 \mathrm{~cm}^{-1}$ ) are due to $\mathrm{N}-\mathrm{H}$ stretch. All other frequencies are below $1600 \mathrm{~cm}^{-1}$. Consequently, the specific heat is within 10 per cent of its asymptotic value (classical limit of $2.32 \mathrm{~J} /(\mathrm{g} \cdot \mathrm{K})$ ) by $1500 \mathrm{~K}$. This is about the temperature behind the von Neumann spike of a CJ detonation wave in PBX 9502. For the lower pressures at which shock initiation occurs, the temperature variation of the specific heat is significant. 
Table 1: Lattice frequencies for TATB based on density functional theory [15, table 2] calculations and Raman spectrum [14, table 1] measurements; see [15] for mode identification. Three acoustic modes not included.

\begin{tabular}{|cc|cc|cc|}
\hline \hline mode & $\begin{array}{c}\text { frequency } \\
\left(\mathrm{cm}^{-1}\right)\end{array}$ & mode & $\begin{array}{c}\text { frequency } \\
\left(\mathrm{cm}^{-1}\right)\end{array}$ & mode & $\begin{array}{c}\text { frequency } \\
\left(\mathrm{cm}^{-1}\right)\end{array}$ \\
\hline- & 51 & Q21 & 611 & Q44 & 1173 \\
- & 62 & Q22 & 656 & Q45 & 1177 \\
- & 80 & Q23 & 660 & Q46 & 1231 \\
- & 97 & Q24 & 804 & Q47 & 1215 \\
- & 109 & Q25 & 691 & Q48 & 1219 \\
- & 119 & Q26 & 730 & Q49 & 1320 \\
- & 133 & Q27 & 768 & Q50 & 1327 \\
- & 139 & Q28 & 704 & Q51 & 1340 \\
- & 148 & Q29 & 708 & Q52 & 1397 \\
- & 231 & Q30 & 683 & Q53 & 1416 \\
Q8 & 292 & Q31 & 740 & Q54 & 1446 \\
Q9 & 295 & Q32 & 744 & Q55 & 1453 \\
Q10 & 312 & Q33 & 779 & Q56 & 1507 \\
Q11 & 318 & Q34 & 821 & Q57 & 1517 \\
Q12 & 370 & Q35 & 827 & Q58 & 1575 \\
Q13 & 371 & Q36 & 837 & Q59 & 1586 \\
Q14 & 391 & Q37 & 860 & Q60 & 1596 \\
Q15 & 388 & Q38 & 870 & Q61 & 3313 \\
Q16 & 436 & Q39 & 874 & Q62 & 3326 \\
Q17 & 438 & Q40 & 1026 & Q63 & 3334 \\
Q18 & 498 & Q41 & 1032 & Q64 & 3426 \\
Q19 & 520 & Q42 & 1148 & Q65 & 3436 \\
Q20 & 521 & Q43 & 1119 & Q66 & 3439 \\
\hline
\end{tabular}




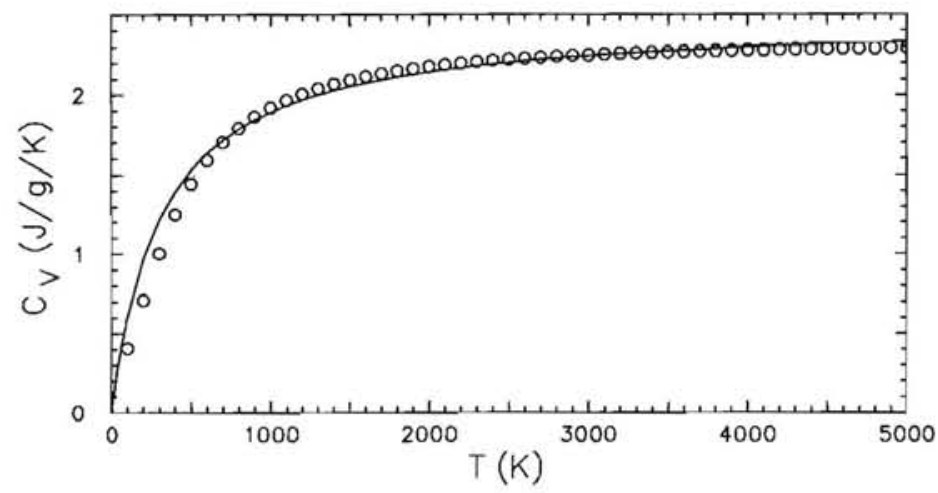

Figure 2: Specific heat for TATB. Symbols are computed points based on lattice vibrational frequencies and line is fit using Eq. (12).

The Grüneisen coefficient is related to the variation of the vibrational frequencies with $V$. Several groups of researchers have studied this dependence at room temperature. Noteworthy are the Raman scattering measurements of low frequency modes at low pressure $(P<0.2 \mathrm{GPa})$ by Holy [16], and the infrared absorption measurements of selected modes up to $12 \mathrm{GPa}$ by Pravica et al. [11]. Over the range of the measurements, the vibrational frequencies display an approximate linear dependence on pressure.

The mode $\Gamma_{i}$ can be expressed as

$$
\Gamma_{i}(V)=K(P(V))\left[\frac{\mathrm{d} \ln \nu_{i}}{\mathrm{~d} P}\right] .
$$

The fits to diamond anvil cell data [9] described in the next subsection determine $K(P)$ and $P(V)$. These functions are shown in fig. 3. This in conjunction with measurements of $\nu_{i}(P)$ enable us to determine the mode $\Gamma_{i}(V)$. Data is only available for a limited number of modes. For those modes, the frequency and the value of $\Gamma_{i}$ are listed in table 2. Not listed are the highest frequencies, $\nu>3200 \mathrm{~cm}^{-1}$, for which the data [11, fig. 4a] displays the anomalous behavior of a negative $\Gamma_{i}$. Pravica et al. [11] attribute this to the intermolecular hydrogen bonding between lattice planes.

For the three acoustic modes, neglecting the crystal anisotropy, we can estimate the mode $\Gamma_{i}$ using the Vashchenko-Zubarev relation (see [17, sec. 3.5] and references therein)

$$
\Gamma_{a}=\frac{1}{2}\left(K_{0}^{\prime}-\frac{5}{3}\right)
$$


Table 2: Mode Grüneisen coefficient for selected frequencies of TATB. Low pressure data from [16, table 2], and high pressure data from [11, figs. 4b \& 6].

\begin{tabular}{|ccc|ccc|}
\hline \multicolumn{3}{c}{ low pressure data } & \multicolumn{3}{c|}{ high pressure data } \\
\begin{tabular}{|ccc|ccc|} 
frequency \\
$\left(\mathrm{cm}^{-1}\right)$
\end{tabular} & $\begin{array}{c}\frac{\mathrm{d} \ln \nu_{i}}{\mathrm{~d} P} \\
\left(\mathrm{GPa}^{-1}\right)\end{array}$ & $\begin{array}{c}\Gamma_{i} \\
(P=0\end{array}$ & $\begin{array}{c}\text { frequency } \\
\left(\mathrm{cm}^{-1}\right)\end{array}$ & $\begin{array}{c}\frac{\mathrm{d} \ln \nu_{i}}{\mathrm{~d} P} \\
\left(\mathrm{GPa}^{-1}\right)\end{array}$ & $@ P=5 \mathrm{GPa}$ \\
\hline 30.69 & 0.3773 & 5.13 & 305 & 0.0079 & 0.42 \\
32.88 & 0.4620 & 6.28 & 455 & 0.0033 & 0.18 \\
47.74 & 0.3137 & 4.27 & 710 & 0.0021 & 0.14 \\
55.86 & 0.2169 & 2.95 & 1460 & 0.0016 & 0.10 \\
99.32 & 0.2072 & 2.82 & & & \\
367.83 & 0.0184 & 0.25 & & & \\
386.26 & 0.0211 & 0.29 & & & \\
\hline
\end{tabular}

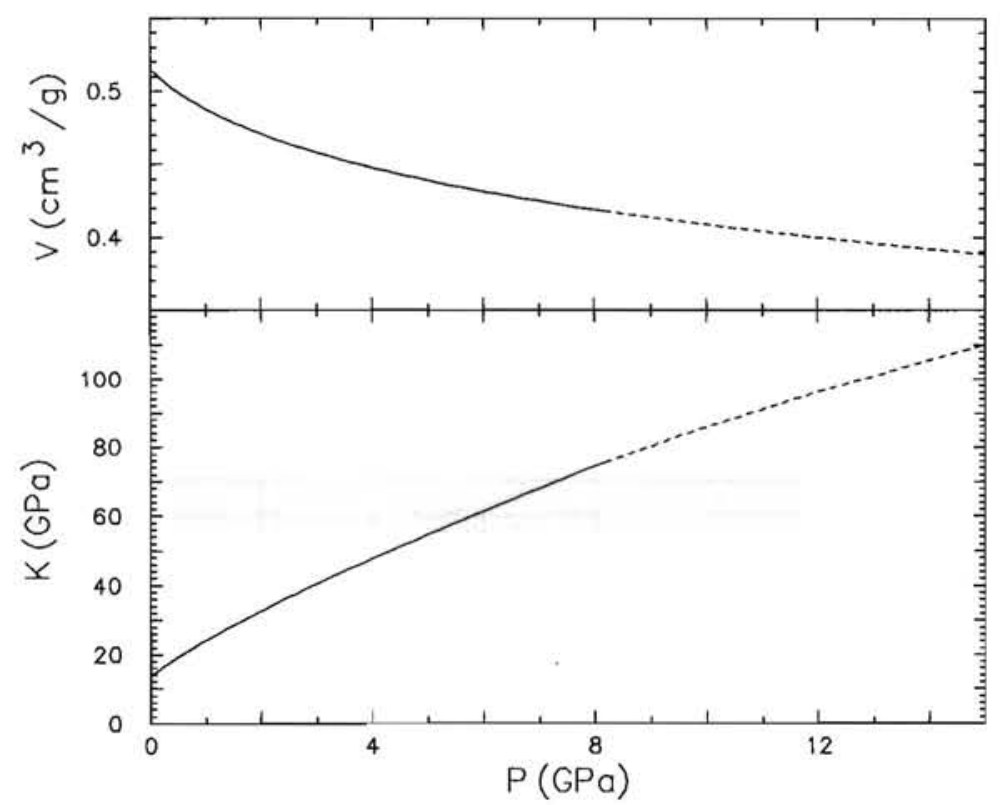

Figure 3: Bulk modulus and specific volume as a function of pressure along cold curve of TATB. Solid curve is based on low pressure Keane fitting parameters and dashed curve based on high pressure parameters. 
Substituting the value $K_{0}^{\prime}=12.4$, from diamond anvil cell experiments discussed in the next subsection, gives $\Gamma_{a}=5.4$. This is comparable to the mode $\Gamma_{i}$ for the low frequency modes listed in table 2 .

Two trends are evident the data. First, the mode $\Gamma_{i}$ decreases with increasing frequency. Second, to the extent that $\frac{d \ln \nu_{i}}{d \ln P}$ is constant, the mode $\Gamma_{i}$ increases with pressure. As a consistency check we note that at the ambient state ( 1 bar and $300 \mathrm{~K}$ ) $\Gamma$ can be determined from the thermodynamic relation

$$
\Gamma_{0}=V_{0} \frac{\beta K_{s}}{C_{p}},
$$

where $\beta$ is the volumetric thermal expansion coefficient, $K_{s}$ is the isothermal bulk modulus, and $C_{p}$ is the specific heat at constant pressure. From [18, pp. 156-157] $\beta=2.36 \times 10^{-4} \mathrm{~K}^{-1}$ and $C_{p}=1.039 \times 10^{-3} \mathrm{MJ} /(\mathrm{kg} \cdot \mathrm{K})$ at $T=25 \mathrm{C}$. Together with $K_{s}=13.6 \mathrm{GPa}$ from fit in next subsection, gives ${ }^{1}$ $\Gamma_{0}=1.6$. Since this value lies between that of the low frequency and high frequency $\Gamma_{i}$, it is compatible with the limited available data.

Given the crude approximation of a single temperature scale model and limited data for the Grüneisen coefficient, we simply take $\Gamma(V)=0.5 \mathrm{~V} / V_{0}$. This has the expected trend that $\Gamma$ decreases with compression. It does not agree with $\Gamma_{0}$ at ambient state. However, near the ambient state the Grüneisen coefficient has a negligible effect, whereas a release to $V_{0}$ from a strong shock would be at a high temperature for which a lower $\Gamma$ is more reasonable.

If and when sufficient data becomes available to determine the mode $\Gamma_{i}$, then the thermal model can be extended to have multiple temperature scales; see [4, sec. 4.3.4]. This would result in $\Gamma$ being a function of both $V$ and $T$. Evaluations of the pressure would be computationally more expensive. If this became burdensome then one could use the model to generate a tabular EOS, such as the SESAME EOS. The advantage of a tabular EOS is that the cost to evaluate the pressure or temperature at a point is independent of the complexity of the model used to generate the table. The trade-off is with the memory requirement for having a fine enough table for an accurate interpolation.

\footnotetext{
${ }^{1}$ Using Eq. (24), Lambourn et al. [3] obtained $\Gamma_{0}=0.58$. The discrepancy is due to differing values of $\beta$ from x-ray diffraction measurments of TATB crystal [19] and from pressed powder dilatometry measurements. In addition, there is an uncertainty in the value of $K_{s}$ at $V_{0}$.
} 


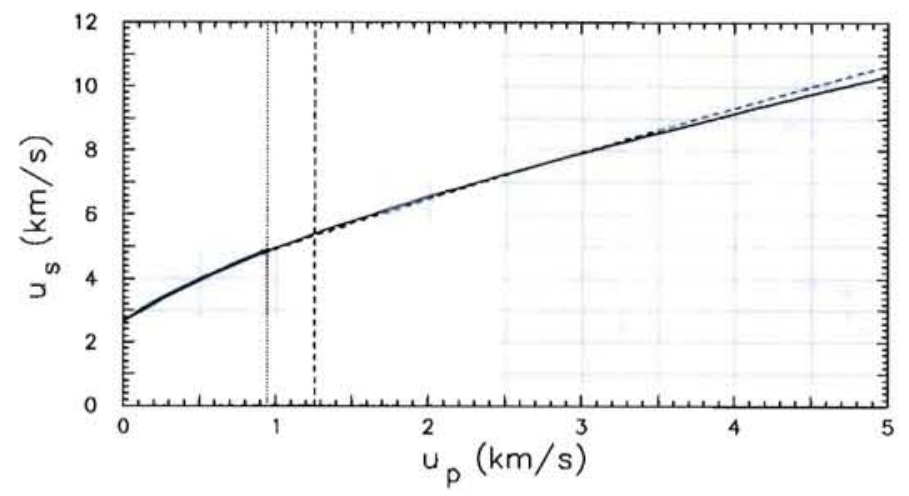

Figure 4: TATB isotherm at room temperature. Black curve is from EOS derived here. Blue curves are fit to diamond anvil cell data using BirchMurnaghan parameters in [9, table 2]; solid curve is fit below $8 \mathrm{GPa}$ and dashed curve is for all data, up to $13.2 \mathrm{GPa}$, and extrapolation beyond; $u_{p}>$ $1.26 \mathrm{~km} / \mathrm{s}$ Dotted black and dashed black lines indicate the extent of the fits.

\subsection{Cold curve}

We calibrate the cold curve parameters to isothermal compression data from diamond anvil cell experiments by Stevens et al. [9]. Two Birch-Murnaghan fits are given in [9]: (i) up to pressure of $8 \mathrm{GPa}$, and (ii) for all data, pressure up to $13.2 \mathrm{GPa}$. The reason for the two fits is that the data [9, fig. 7] displays a kink at a pressure of about $8 \mathrm{GPa}$. This in part motivates our choice of a two-piece Keane fitting form.

A comparison of the model EOS with the data, in the pseudo-velocity plane, is shown in fig. 4. The computed curve utilized the specific heat and the Grüneisen coefficient discussed in the previous subsection to obtain the room temperature isotherm, $T=300 \mathrm{~K}$. The data only extends up to $u_{p}=1.26 \mathrm{~km} / \mathrm{s}$. Beyond that the experimental curve represents an extrapolation. The plot extends up to $u_{p}=5 \mathrm{~km} / \mathrm{s}$. This corresponds to the limit of overdriven detonation wave data, roughly a pressure of $100 \mathrm{GPa}$. The parameters of the second Keane fit were selected in part such that the Hugoniot locus and detonation locus do not intersect. This is discussed further in the next subsection. For now we note that there is a slight difference with the extrapolated fit to the isothermal data and the EOS model at pressures well beyond that of the highest data point. 


\section{PBX 9502 model EOS}

For the porosity model, the solid volume fraction $\phi_{0}$ is determined by the ratio of the initial PBX 9502 density to the theoretical maximum density. For the crush-up pressure we chose $P_{c r}=0.4 \mathrm{GPa}$. This only affects the Hugoniot locus at low pressures; mainly the initial sound speed which is the intercept of the locus in the $\left(u_{p}, u_{s}\right)$-plane.

By way of summary, the EOS parameters for the complete PBX 9502 model are listed in table 4.

\subsection{Comparison with Hugoniot locus}

As a check on the PBX 9502 EOS model we compare with Hugoniot data of Dick et al. [10] and Gustavsen et al. [20]. Though our focus is on the reactant EOS it is also helpful to plot the overdriven detonation locus. We use the data from Tang et al. [21] and Green et al. [22], and a SESAME EOS table for the products generated by Sam Shaw and briefly described in [23, App. D].

To set the scale, key quantities of a CJ detonation wave in PBX 9502 are listed in table 3. The Hugoniot locus and detonation locus are shown in the $(V, P)$-plane and $\left(u_{p}, u_{s}\right)$-plane in figs. 5 and 6 , respectively. Considering the scatter in the data, the agreement with the model is as good as can be expected.

Table 3: CJ detonation wave in PBX 9502.

\begin{tabular}{|l|ccccc|}
\hline \hline & $\begin{array}{c}V \\
\mathrm{~cm}^{3} / \mathrm{g}\end{array}$ & $\begin{array}{c}e \\
\mathrm{MJ} / \mathrm{kg}\end{array}$ & $\begin{array}{c}P \\
\mathrm{GPa}\end{array}$ & $\begin{array}{c}T \\
\mathrm{~K}\end{array}$ & $\begin{array}{c}u_{p} \\
\mathrm{~km} / \mathrm{s}\end{array}$ \\
\hline Init state & 0.529 & 0.223 & 0.0001 & 300 & 0.0 \\
\hline \multicolumn{7}{c}{ detonation speed } & $7.73 \mathrm{~km} / \mathrm{s}$ \\
\hline VN spike & 0.328 & 4.516 & 42.8 & 1567 & 2.93 \\
CJ state & 0.396 & 2.100 & 28.3 & 3126 & 1.94 \\
\hline
\end{tabular}


Table 4: EOS parameters for PBX 9502.

\begin{tabular}{l}
\hline \hline TATB reference state \\
\begin{tabular}{|l|cl|}
\hline$V_{0}$ & $1 / 1.942$ & $\mathrm{~cm}^{3} / \mathrm{g}$ \\
$P_{0}$ & -0.216328 & $\mathrm{GPa}$ \\
$e_{0}$ & 0.0 & $\mathrm{MJ} / \mathrm{kg}$ \\
\cline { 2 - 3 }$e_{*}$ & 0.222892 & $\mathrm{MJ} / \mathrm{kg}$ \\
$P\left(V_{0}, e_{*}\right)$ & 0.0001 & $\mathrm{GPa}$ \\
$T\left(V_{0}, e_{*}\right)$ & 300. & $\mathrm{~K}$ \\
\hline
\end{tabular}
\end{tabular}

TATB cold curve

\begin{tabular}{|l|ll|}
\hline$K_{0}$ & 13.6 & $\mathrm{GPa}$ \\
$K_{0}^{\prime}$ & 12.4 & - \\
$K_{0, \infty}^{\prime}$ & 5.0 & - \\
$V_{1} / V_{0}$ & 0.79 & - \\
$K_{1}^{\prime}$ & 6.0 & - \\
$K_{1, \infty}^{\prime}$ & 1.001 & - \\
\hline
\end{tabular}

TATB Grüneisen coefficient \& $\theta$

\begin{tabular}{|l|ll|}
\hline$\Gamma_{0}$ & 0.0 & - \\
$\Gamma_{1}$ & 0.5 & - \\
$\theta_{0}$ & 1.0 & $\mathrm{~K}$ \\
$\epsilon_{1}$ & 0.3 & - \\
\hline
\end{tabular}

TATB specific heat

\begin{tabular}{|l|ll|}
\hline$C_{v}$ & $1.0 \times 10^{-3}$ & $\mathrm{MJ} /(\mathrm{kg} \cdot \mathrm{K})$ \\
$c_{0}$ & $4.480 \times 10^{-4}$ & - \\
$c_{1}$ & $2.229 \times 10^{-1}$ & - \\
$c_{2}$ & $1.256 \times 10^{2}$ & - \\
$c_{3}$ & $4.034 \times 10^{-1}$ & - \\
\hline
\end{tabular}

porosity parameters

\begin{tabular}{|l|ll|}
\hline$\phi_{0}$ & 0.9732 & - \\
$P_{\text {cr }}$ & 0.4 & $\mathrm{GPa}$ \\
\hline
\end{tabular}




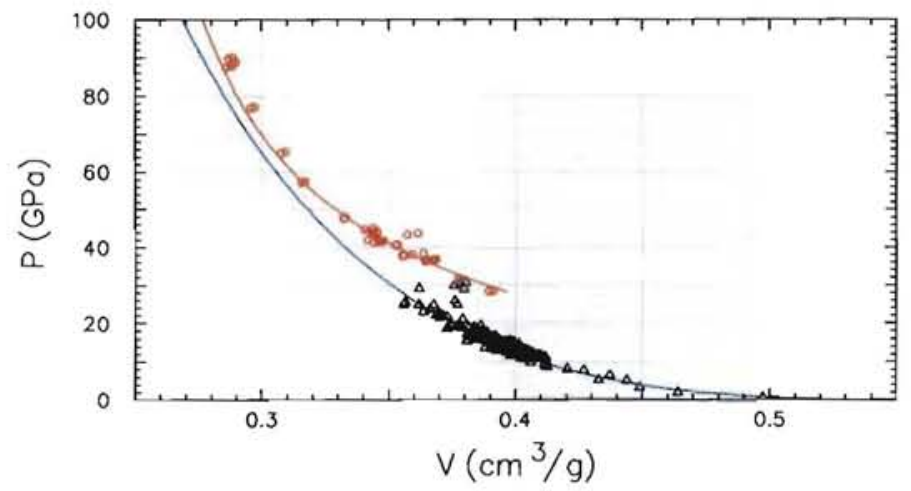

Figure 5: Hugoniot loci for PBX 9502 in $(V, P)$-plane. Black symbols are unreacted shock wave data. Red symbols are overdriven detonation wave data. Blue curve is shock locus calculated with the reactant EOS model. Red curve is the detonation locus calculated with a SESAME EOS for the products.

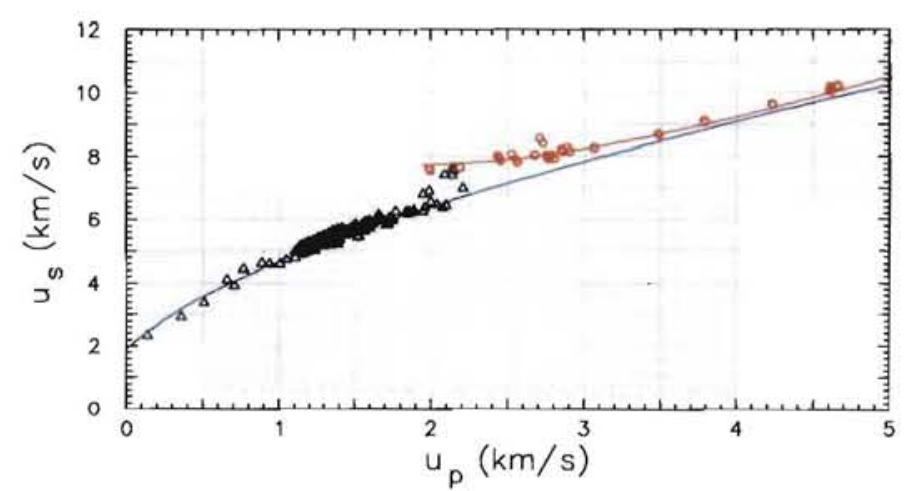

Figure 6: Hugoniot loci for PBX 9502 in $\left(u_{p}, u_{s}\right)$-plane. Black symbols are unreacted shock wave data. Red symbols are overdriven detonation wave data. Blue curve is shock locus calculated with the reactant EOS model. Red curve is the detonation locus calculated with a SESAME EOS for the products. 
We note that at high pressures the shock locus is very close to the detonation locus. We do not expect the loci to cross below the von Neumann spike pressure. For sufficiently strong shocks, the large shock compression can change the electron distribution around the atoms and lower the potential barrier. If the barrier drops below the thermal energy from the shock temperature, then the reactants would be thermodynamically unstable and spontaneously decompose. If this were to occur then the loci may very well cross. It is not known whether this occurs or if so, what a sufficiently large pressure for the transition would be. We chose to set the Keane cold curve parameters such that the loci do not cross. The parameters $K_{1}^{\prime}$ and $K_{1, \infty}^{\prime}$ give sufficient flexibility to adjust the asymptotic behavior if additional very high pressure data should become available. We also note that the weak discontinuity in $K$ where the the two Keane fits join together is not noticeable in the Hugoniot locus in either plane, nor in the plot of $K(P)$ in fig. 3.

Comparing with another model gives some perspective on the sensitivity of the EOS to fitting forms. One of the better models for PBX 9502 is by Wescott et al. [24]. In addition, they used the same Hugoniot data to calibrate model parameters. Figures 7 and 8 show the computed Hugoniot loci for both models. The locus in the $\left(u_{p}, u_{s}\right)$-plane reflect the pressure component of the EOS. It can be seen that this part of the EOS is pretty much the same within the range of the data; up to about $25 \mathrm{GPa}$. The models begin to differ when used to extrapolate to higher shock pressures. But even at $100 \mathrm{GPa}$ they are within 4 per cent. With neither data nor theory at high pressures, there is no basis to prefer one or the other.

The models do differ in their thermal properties; $C_{V}$ which affects the shock temperature as seen in the loci in the $(P, T)$-plane shown in fig. 8 . Though there is no temperature data, there is a theory for the specific heat, which is described in sec. 5 , and spectral data to calibrate it. Closely related to the specific heat is the Grüneisen coefficient which affects the pressure off the principal Hugoniot locus. Both models make the simplifying assumption that $\Gamma$ depends on only $V$. The spectral data indicates that this assumption is not correct. For the most part, the compressional pressure is much larger than the thermal pressure and hydro simulations are not too sensitive to the Grüneisen coefficient.

Figure 8 shows the temperature on both the shock locus and detonation locus. We note that there is over a factor of 2 difference between the unreacted temperature at the von Neuman spike and the CJ temperature of the 


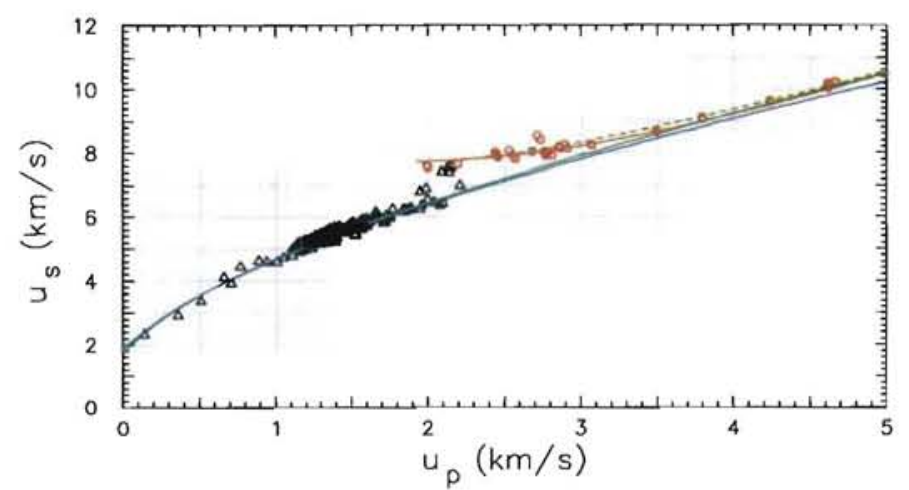

Figure 7: Comparison of PBX 9502 Hugoniot loci in $\left(u_{p}, u_{s}\right)$-plane. Black and red points and curves are as in fig. 6. In addition, green curves are computed from EOS model of Wescott et al. [24]; solid line is shock locus and dashed line is detonation locus.

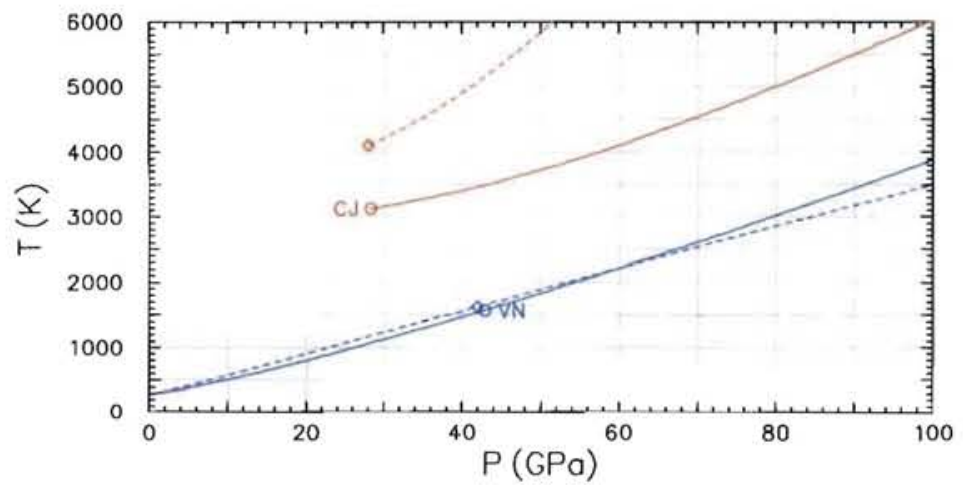

Figure 8: Comparison of PBX 9502 Hugoniot loci in $(P, T)$-plane. Black and red solid curves are shock locus and detonation locus, respectively, computed from reactant EOS model and SESAME EOS for products. Corresponding dash curves are computed from EOS model of Wescott et al. [24]. 
products. The ratio is much larger than for the more potent explosive HMX. This ratio may affect the homogenized reaction rate from hot-spot grow, and consequently detonation properties of a plastic-bonded explosive; in particular, sensitivity of shock initiation to the grain-size distribution.

\subsection{Hugoniot loci for different initial densities}

By changing only 1 parameter, $\phi_{0}$, the porosity model allows the initial density of the PBX to be varied. Figures 9 and 10 shows the effect of varying the density on the principal Hugoniot locus. In the $\left(u_{p}, u_{s}\right)$-plane, the loci shift down with increasing porosity, which implies an increased compression for the same shock pressure. In the $(P, S)$-plane, the loci shift up with increasing porosity, which implies increased shock heating.

Porosity also increases the sensitivity to shock initiation. The CREST model $[2,25]$ captures this effect, and also shock desensitization, by taking the reaction rate to be a function of the reactant entropy. Possibly this can be interpreted as the reaction rate is proportional to the number or size of hot spots which scales with the shock dissipation. The entropy increase is a measure of shock dissipation. For such a model, thermal properties are important since $S$ and $T$ are conjugate thermodynamic variables.

\section{Summary}

A complete EOS model for the reactants of PBX 9502 has been constructed based on the available data. This includes isothermal compression data of TATB, the Raman and infrared spectrum of TATB and shock data for unreacted PBX 9502. The model is thermodynamically consistent and enables the initial PBX density to be varied by adjusting its initial porosity.

Both the pressure and temperature should be reasonably accurate on the principal shock locus. Off the shock locus, simplifying assumptions and the choice of fitting forms affect the accuracy of the EOS. There are three main limitations to the model:

(i) The model assumes that the Grüneisen coefficient is a function of only $V$, while spectral data show multiple temperature scales for the mode $\Gamma_{i}$ of the lattice vibrations. This implies that $\Gamma$ is a function of both $V$ and $T$. We 


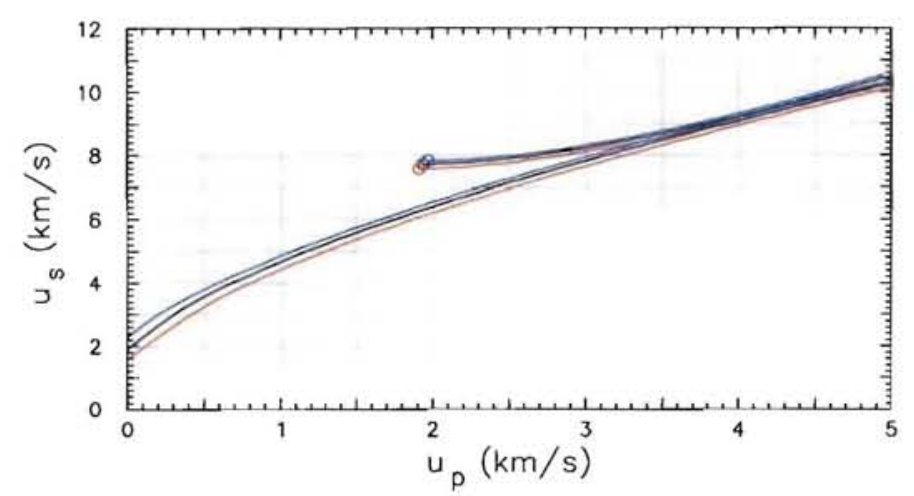

Figure 9: Variation of PBX 9502 Hugoniot loci in $\left(u_{p}, u_{s}\right)$-plane with porosity. Black curve is standard porosity, $2.4 \%$. Blue curve is lower porosity, $1 \%$, and red curve is higher, $5 \%$.

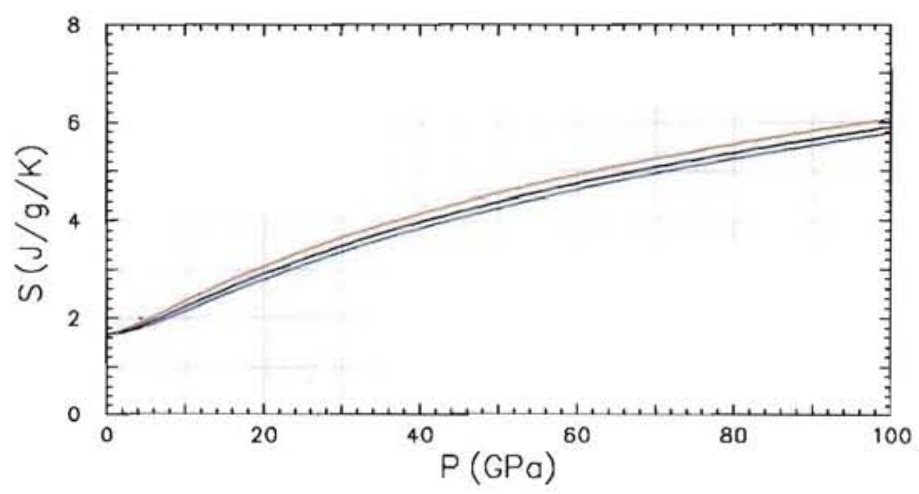

Figure 10: Variation of PBX 9502 Hugoniot loci in $(P, S)$-plane with porosity. Black curve is standard porosity, $2.4 \%$. Blue curve is lower porosity, $1 \%$, and red curve is higher, $5 \%$. 
expect that $\Gamma$ will decrease as $T$ increases above the shock locus, i.e., for large $T$ the thermal pressure would be too large.

(ii) The fitting form for the cold curve $P_{c}(V)$ allows extrapolation to higher pressures than the shock data, i.e., $P>25 \mathrm{GPa}$. The further one extrapolates the larger the uncertainty.

(iii) The expansion region, $\rho<\rho_{0}$, is well behaved but arbitrary. The expansion region is not accurate. It is included for robustness when the EOS is used in hydro simulation.

\section{Acknowledgements}

The author thanks Tariq Aslam for suggesting the use of the Keane EOS as a fitting form and for providing an electronic file with the PBX 9502 Hugoniot data, Shawn McGrane for discussions on the Raman spectrometry, and M. Sam Shaw for providing a SESAME table for PBX 9502 product and discussions on EOS and TATB.

\section{References}

[1] E. L. Lee and C. M. Tarver. Phenomenological model of shock initiation in heterogeneous explosives. Phys. Fluids, 23:2362-2372, 1980.

[2] C. A. Handley. The CREST reactive burn model. In Thirteenth Symposium (International) on Detonation, pages 864-870, 2006.

[3] B. D. Lambourn, N. J. Whitworth, C. A. Handley, and H. R. James. A finite strain, non-reactive EOS for PBX 9502. In Shock Compression of Condensed Matter - 2007, pages 137-140, 2008.

[4] R. Menikoff. Empirical equations of state for solids. In Y. Horie, editor, Solids I, volume 2 of Shock Wave Science and Technology Reference Library, chapter 4. Springer-Verlag, 2007.

[5] R. Menikoff. Generalized Hayes equation of state. Technical Report LA-UR-08-06984, Los Alamos National Laboratory, 2008.

[6] R. Menikoff and T. D. Sewell. Complete equation of state for beta-HMX and implications for initiation. In Shock Compression of Condensed Matter - 2003, pages 157-160, 2004.

[7] J. Shanker and B. P. Singh. A comparative study of Keane's and Stacey's equation of state. Physica B, 370:78-83, 2005. 
[8] Y. X. Zhao and I. L. Spain. X-ray diffraction data for graphite to 20 GPa. Phys. Rev. B, 40:993-997, 1989.

[9] L. L. Stevens, N. Velisavljevic amd D. E. Hooks, and D. M. Dattelbaum. Hydostatic compression curve for Triamino-Trinitrobenzene determined up to $13 \mathrm{GPa}$ with powder X-ray diffraction. Propellants, Explosives, Pyrotechnics, 33:286-295, 2008.

[10] J. J. Dick, C. A. Forest, J. B. Ramsay, and W. L. Seitz. The Hugoniot and shock sensitivity of a plastic-bonded TATB explosive PBX 9502. J. Appl. Phys, 63:4881-4888, 1988.

[11] M. Pravica, B. Yulga, Z. Liu, and O. Tschauner. Infrared study of 1,3,5-triamino-2,4,6-trinitrobenzene under high pressure. Phys. Rev. B, 76:064102/4, 2007.

[12] M. F. Foltz. Behavior of explosives under pressure in a diamond anvil cell. In Thirteenth Symposium (International) on Detonation, pages 997-1006, 2006.

[13] R. Menikoff and E. Kober. Equation of state and Hugoniot locus for porous materials: $p-\alpha$ model revisited. In Shock Compression of Condensed Matter - 1999, pages 129-132, 2000.

[14] S. D. McGrane and A. P. Shreve. Temperature-dependent Raman spectra of triaminotrinitrobenzene: Anharmonic mode couplings in an energetic material. J. Chem. Phys., 119:5834-5841, 2003.

[15] H. Liu, J. Zhao, G. Ji, D. Wei, and Z. Gong. Vibrational properties of molecule and crystal of TATB: A comparative density functional study. Physics Letters A, 358:63-69, 2006.

[16] J. Holy. Raman spectrocopy of animated and ultrafine 1,3,5-triamino2,4,6-trinitrobenzene and PBX 9502 as a function of pressing pressure. J. Phys. Chem. B, 112:7489-7498, 2008.

[17] J.-P. Poirier, editor. Introduction to the Physics of the Earth's Interior. Cambridge Univ. Press, 1991.

[18] T. R. Gibbs and A. Popolato, editors. LASL Explosive Property Data. Univ. of Calif. Press, 1980. URL http://1ib-www.1anl.gov/ladcdmp/ epro.pdf.

[19] J. R. Kolb and H. F. Rizzo. Growth of 1,3,5-triamino-2,4,6trinitrobenzene (TATB) I. anisotropic thermal expansion. Propellants and Explosives, 4:10-16, 1979.

[20] R. L. Gustavsen, S. A. Sheffield, and R. R. Alcon. Measurements of shock initiation in the tri-amino-tri-nitro-benzene based explosive PBX 9502: Wave forms from embedded gauges and comparison of four dif- 
ferent material lots. J. Appl. Phys, 99:114907/17, 2006.

[21] P. K. Tang, W. W. Anderson, J. N. Fritz, R. S. Hixson, and J. E. Vorthman. A study of the overdriven behaviors of PBX 9501 and PBX 9502. In Eleventh Symposium (International) on Detonation, pages 1058-1064, 1998.

[22] L. Green, E. Lee, A. Mitchell, and C. Tarver. The supra-compression of LX-07, LX-17, PBX-9404, and RX-26-AF and the equation of state of the detontion products. In Eigthth Symposium (International) on Detonation, pages 587-595, 1985.

[23] T. N. Dey and Shaw M. S. A coupled pseudo-reaction zone and products eos model for pbx 9502. Technical Report LA-UR-05-7511, Los Alamos National Laboratory, 2005.

[24] B. L. Wescott, D. S. Stewart, and W. C. Davis. Equation of state and reaction rate for condensed-phase explosives. J. Appl. Phys., 98: 053514/10, 2005.

[25] C. A. Handley and B. D. Lambourn. Predicting the effect of pososity on the shock sensitivity of explosives. In Shock Compression of Condensed Matter - 2009, 2010. 